\title{
Aortic-Brachial Pulse Wave Velocity Ratio: A Measure of Arterial Stiffness Gradient Not Affected by Mean Arterial Pressure
}

\author{
Catherine Fortier Marie-Pier Desjardins Mohsen Agharazii \\ CHU de Québec Research Center, L'Hôtel-Dieu de Québec Hospital, and Division of \\ Nephrology, Faculty of Medicine, Université Laval, Québec, QC, Canada
}

\section{Keywords}

Aortic stiffness · Arterial stiffness · Arterial stiffness gradient · Pulse wave velocity · Blood pressure

\begin{abstract}
Background: Aortic stiffness, measured by carotid-femoral pulse wave velocity (cf-PWV), is used for the prediction of cardiovascular risk. This mini-review describes the nonlinear relationship between cf-PWV and operational blood pressure, presents the proposed methods to adjust for this relationship, and discusses a potential place for aortic-brachial PWV ratio (a measure of arterial stiffness gradient) as a blood pressure-independent measure of vascular aging. Summary: PWV is inherently dependent on the operational blood pressure. In crosssectional studies, PWV adjustment for mean arterial pressure (MAP) is preferred, but still remains a nonoptimal approach, as the relationship between PWV and blood pressure is nonlinear and varies considerably among individuals due to heterogeneity in genetic background, vascular tone, and vascular remodeling. Extrapolations from the blood pressure-independent stiffness parameter $\beta\left(\beta_{0}\right)$ have led to the creation of stiffness index $\beta$, which can be used for local stiffness. A similar approach has been used for cardio-ankle PWV to generate a blood pressure-independent cardio-ankle vascular index (CAVI). It was recently demonstrated that stiffness index $\beta$ and CAVI remain slightly blood pressure-dependent, and a more appropriate formula has been proposed to make the proper adjustments. On the other hand, the negative impact of aortic stiffness on clinical outcomes is thought to be mediated through attenuation or reversal of the arterial stiffness gradient, which can also be influenced by a reduction in peripheral medium-sized muscular arteries in conditions that predispose to accelerate vascular aging. Arterial stiffness gradient, assessed by aortic-brachial PWV ratio, is
\end{abstract}

Mohsen Agharazii, MD

Service de Néphrologie, CHU de Québec

L'Hôtel-Dieu de Québec, 11, Côte du Palais

Québec, QC G1R 2J6 (Canada)

E-Mail Mohsen.Agharazii @ crhdq.ulaval.ca 
emerging to be at least as good as cf-PWV for risk prediction, but has the advantage of not being affected by operating MAP. Key Messages: The negative impacts of aortic stiffness on clinical outcomes are proposed to be mediated through attenuation or reversal of arterial stiffness gradient. Aortic-brachial PWV ratio, a measure of arterial stiffness gradient, is independent of MAP.

(c) 2017 S. Karger AG, Basel

\section{Introduction}

Aortic stiffness is an independent predictor of cardiovascular events and mortality in the general population, and in various pathological conditions such as hypertension, diabetes, and end-stage renal disease [1-4]. Despite its inherent pressure dependency, aortic stiffness assessed by carotid-femoral pulse wave velocity (cf-PWV) is widely used as a noninvasive and reliable clinical tool for cardiovascular risk classification [5]. With aging, the aorta loses its elasticity and its capacity to dampen the pulsatile pressure leading to increased myocardial workload. However, the stiffness of medium-sized muscular arteries was traditionally considered to be relatively unchanged over a lifetime. This knowledge has successfully been used to develop more user-friendly devices to assess vascular stiffness for large epidemiological studies. However, most of these devices rely on a global measure of arterial stiffness, which integrates a combination of both central and peripheral arterial stiffness. In light of reports stating that peripheral stiffness may decrease with age or under pathological conditions [6-8], it might be desirable to further explore this heterogeneity of the arterial tree. Indeed, a decrease in peripheral arterial stiffness could partially contribute to the attenuation or reversal of the stiffness gradient, inducing higher pulse pressure transmission into the microcirculation, causing end-organ damages. In this review, we will discuss (1) the dependency of PWV on the operating blood pressure, (2) the approaches that are proposed to adjust for blood pressure, (3) the use of aortic-brachial PWV ratio as a measure of arterial stiffness gradient, and (4) the relationship between aortic-brachial PWV ratio and blood pressure.

\section{PWV and Operating Blood Pressure}

The Bramwell-Hill variant of the Moens-Korteweg equation states that:

$$
P W V=\sqrt{\frac{d P \times V}{\rho \times d V}},
$$

where $d P$ is change in pressure, $d V$ is change in arterial volume, $V$ is the baseline volume of the vessel, and $\rho$ is blood density. For example, the increase in pressure from diastolic pressure to systolic pressure during a single cardiac cycle could result in a variation of $0.7-4 \mathrm{~m} / \mathrm{s}$ of PWV (i.e., without any change in vascular wall property) $[9,10]$. This variability is mainly caused by the organization of elastin, collagen, and vascular smooth muscle cells of the media. Due to passive mechanical properties, at low pressure the load is carried by elastin, while at high pressure the load is carried by stiffer collagen fibers $[11,12]$.

The pressure-diameter relationship varies greatly among individuals due to differences in genetic background, vascular smooth muscle tone $[13,14]$, and vascular wall remodeling related to exposure to cardiovascular risk factors. As arteries stiffen, an upward shift of the pressure-diameter relationship occurs, meaning that higher pressures are required to induce a similar change in diameter (Fig. 1). Indeed, a steeper slope in pressure-diameter rela- 
Fig. 1. Stress-strain relationship. Nonlinear relationship between arterial stress (pressure) and strain (relative change in diameter), with arterial wall components under tension according to the pressure load in normal and stiff vessels. Reprinted from McEniery et al. [40] with permission.

Fortier et al.: Aortic-Brachial Pulse Wave Velocity Ratio: A Measure of Arterial Stiffness Gradient Not Affected by Mean Arterial Pressure

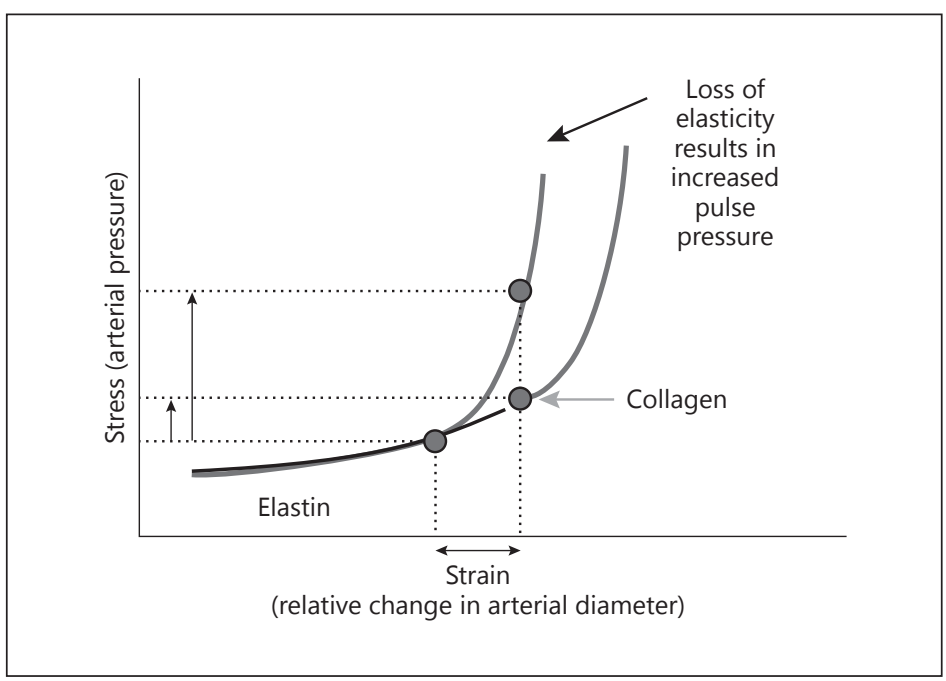

tionship is observed in the elderly compared to younger individuals as pressure dependence varies with age, due partly to the age-related material fatigue, fractures in the elastin lamella, and vascular fibrosis $[9,10,15]$.

\section{Adjusting Arterial Stiffness for Operating Blood Pressure}

In cross-sectional studies, PWV adjustments for pressure are usually performed by a linear regression using the average mean arterial pressure (MAP) (nonpulsatile component of blood pressure) of the cohort. While this method has its merits, it also has its inherent limitations as it does not consider the interindividual differences in PWV-pressure relationship, and it does not take into account the nonlinear nature of this relationship.

Arterial stiffness index $\beta$ and cardio-ankle vascular index (CAVI) theoretically consider the effect of blood pressure, since both are based on the substitution of the exponential pressure-diameter relationship to a linear relationship between the change of pressure (ln $\left(\mathrm{P}_{\mathrm{s}} / \mathrm{P}_{\mathrm{d}}\right)$ ) and relative changes in diameter (Fig. 2) [16-22]. However, stiffness index $\beta$ is obtained by an approximation of the exponential pressure-diameter relationship first described experimentally by Hayashi et al. [23]

$$
P=P_{\text {ref }} e^{\beta 0\left[\left(\frac{d}{d_{r e f}}\right)-1\right]},
$$

from which the pressure and diameter of reference ( $P_{\text {ref }}$ and $\left.d_{\text {ref }}\right)$ were substituted by diastolic pressure and diastolic diameter. Accordingly, the stiffness index $\beta$ is slightly different than the proposed blood pressure-independent $\beta_{0}$. Indeed, Spronck et al. [24] recently demonstrated that arterial stiffness index $\beta$ and CAVI are slightly blood pressure-dependent, and they proposed using a fixed reference pressure value to resolve this issue for local or segmental stiffness.

However, applying such correction to a global PWV that encompasses various heterogeneous vascular territories and treating them as homogenous does not seem to be conceptually appealing.

Indeed, this is clearly demonstrated by Shimizu et al. [25] who examined the impact of acute administration of nitroglycerin on CAVI. Their findings showed a reduction in CAVI 
Fortier et al.: Aortic-Brachial Pulse Wave Velocity Ratio: A Measure of Arterial Stiffness Gradient Not Affected by Mean Arterial Pressure

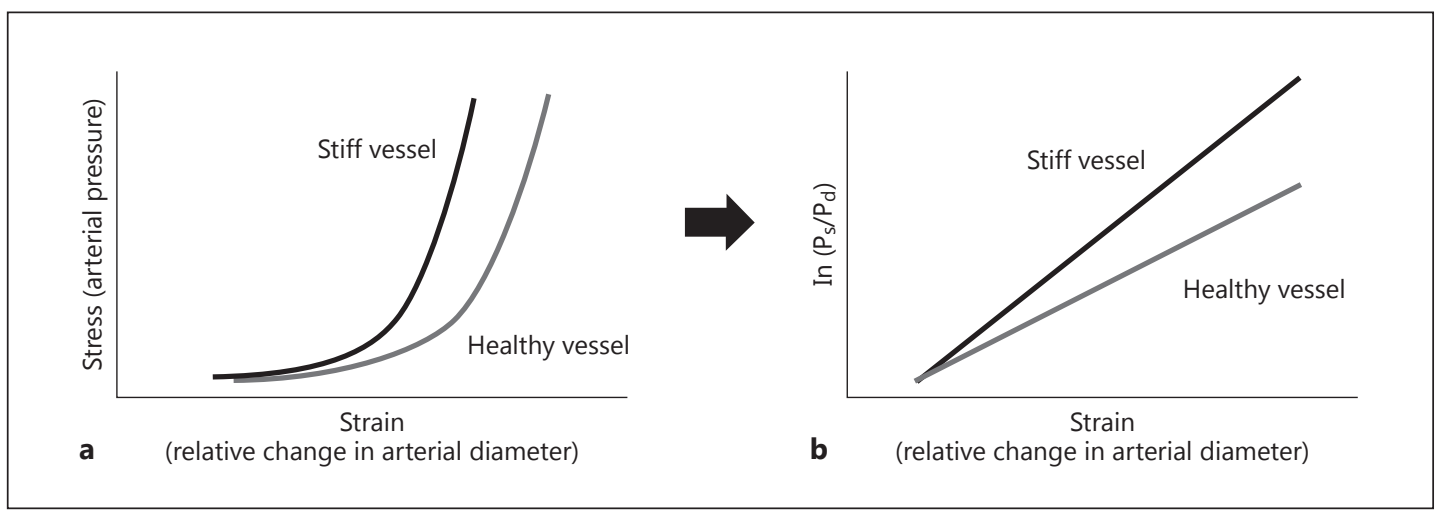

Fig. 2. Pressure-diameter relationship and stiffness index $\beta$. a The exponential pressure-diameter relationship in healthy and stiff vessel. $\mathbf{b}$ The transformation of this relationship into a linear model where the slope represents the proposed blood pressure-independent stiffness index $\beta$.

suggesting an improvement of vascular stiffness. Since CAVI is based on cardio-ankle PWV, it was impossible to evaluate whether this improvement was the result of a reduction in aortic stiffness, femoral-tibial stiffness, or both. Taking this idea further, the same group addressed this question by applying the stiffness index $\beta$ to heart-thigh PWV and thigh-ankle PWV by utilizing Bramwell-Hill's equation in the same way as it is applied when measuring CAVI [26]. They concluded that the reduction of CAVI after nitroglycerin administration was mainly the result of a reduction in thigh-ankle stiffness index $\beta$ rather than a change in the heart-thigh stiffness index $\beta$. Similarly, we observed a significant reduction in carotid-radial PWV (cr-PWV) and femoral-tibial PWV (muscular conduit vessels) without any changes in cf-PWV or MAP [27].

Taken together, these results suggest that the reduction of the stiffness of muscular conduit vessels, which link aorta to the microcirculation, results in the attenuation of arterial stiffness gradient. Given that the loss or reversal of arterial stiffness gradient is proposed to cause microvascular damage, especially to the kidneys and the brain, such effect could theoretically be undesirable.

\section{Aortic-Brachial PWV Ratio: A Measure of Arterial Stiffness Gradient}

Aorta and peripheral medium-sized muscular conduit vessels are structurally and biomechanically different, and may not be similarly affected by age, pathological conditions, and vasoactive drugs $[6,7,26-31]$. Accordingly, arterial stiffness gradient is not only affected by an increase in aortic stiffness, butalso by a decrease in the stiffness of medium-sized peripheral muscular arteries [32-34]. Indeed, in a longitudinal study, we discovered that brachial PWV, as measured by $\mathrm{cr}-\mathrm{PWV}$, decreased by approximately $0.66 \mathrm{~m} / \mathrm{s} / \mathrm{year}$, despite an accelerated increase in cf-PWV by $0.84 \mathrm{~m} / \mathrm{s} /$ year. This divergent evolution of these two vascular territories led us to propose that aortic-brachial PWV ratio (cf-PWV/cr-PWV), a measure of arterial stiffness gradient, could be used to evaluate the risk of mortality. This hypothesis was tested in a cohort of 310 dialysis patients, and it was shown that aortic-brachial PWV ratio predicted mortality better than cf-PWV alone, and was the only significant hemodynamic parameter that resisted multivariable adjusted models [35].

More recently, PWV ratio was assessed in the Framingham Heart Study Offspring cohort, a large general population free of overt cardiovascular disease. It was shown that PWV ratio 
Fortier et al.: Aortic-Brachial Pulse Wave Velocity Ratio: A Measure of Arterial Stiffness Gradient Not Affected by Mean Arterial Pressure

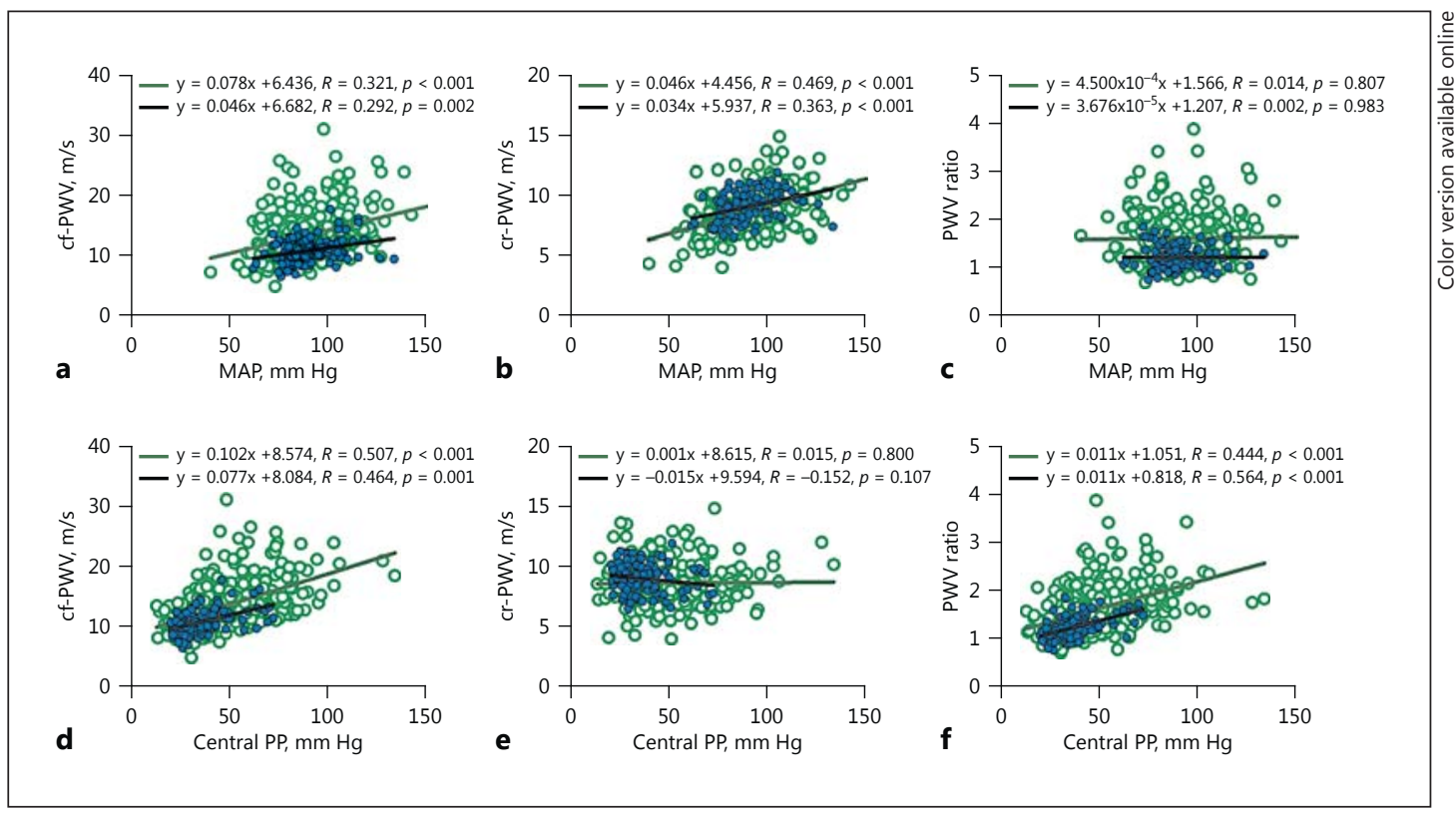

Fig. 3. Aortic stiffness, brachial stiffness and arterial stiffness gradient relationships with mean arterial pressure (MAP) and central pulse pressure (PP). There was a positive relationship between MAP and carotidfemoral pulse wave velocity (cf-PWV, a) and carotid-radial pulse wave velocity (cr-PWV, b), but no relationship between MAP and PWV ratio (c) for the dialysis cohort ( $n=304$, empty circles) and for the cohort with eGFR $>45$ ( $n=114$, filled circles). The relationship between central PP, cf-PWV (d) and PWV ratio (f) were similar in both respective cohorts, while there was no relationship between central PP and cr-PWV (e).

predicts cardiovascular events as well as aortic stiffness, but did not provide any additional risk information above and beyond cf-PWV [36]. This is not surprising before the fifth or sixth decade of age as stiffness of peripheral medium-sized muscular arteries are negligibly affected by age, and the main driving factor for alteration of arterial stiffness gradient is increased aortic stiffness. However, authors mentioned that they could not exclude the possibility that PWV ratio might provide an incremental predictive value over cf-PWV in the elderly, as the association between CV events and PWV ratio was greater than that of aortic stiffness in individuals over the age of 70 . Moreover, other studies tend to support that arterial stiffness gradient may be better associated with target-organ damage than aortic stiffness. For example, in a cross-sectional analysis of patients with type 2 diabetes $(n=60)$ and age-matched controls $(n=60)$, aortic-brachial stiffness gradient predicted estimated glomerular filtration rate independently of age, sex, diabetes status, and cardiovascular risk factors, whereas aortic stiffness did not [37]. In addition, Lee et al. [38] observed that PWV ratio was independently associated with history of stroke and coronary artery disease in patients with a known medical history of cardiovascular disease $(n=142)$, while aortic stiffness did not reach a statistical level of significance.

\section{PWV Ratio and Its Relation to Blood Pressure}

Since aortic and brachial PWVs may both change in the same direction as the operating MAP, we hypothesized that PWV ratio may potentially be blood pressure-independent. We tested this hypothesis in two distinct cohorts of patients that were completely different in 
Fortier et al.: Aortic-Brachial Pulse Wave Velocity Ratio: A Measure of Arterial Stiffness Gradient Not Affected by Mean Arterial Pressure

terms of age, renal function, and comorbidities [7]. In the first group $(n=304)$, which included dialysis patients, aortic stiffness increased with age, while brachial stiffness decreased. In the second group of patients with an estimated glomerular filtration rate of $>45 \mathrm{~mL} / \mathrm{min} / 1.73 \mathrm{~m}^{2}$ $(n=114)$, aortic stiffness increased with age as expected, while brachial stiffness increased slightly until the fifth decade and then started to decrease thereafter. Nevertheless, aortic and brachial stiffness both increased with higher MAP (measured at the time of measurement of vascular stiffness) in the two groups, while there was no correlation between PWV ratio and MAP (Fig. 3a-c). As shown in Figure 3d-f, there was a positive and similar relationship between central pulse pressure, cf-PWV, and PWV ratio, whereas there was no significant relationship between central pulse pressure and cr-PWV.

Similar results have also been observed by other investigators [37, 39]. Picone et al. [37] assessed the determinants of PWV ratio in a diabetic group and a nondiabetic control group. They found that PWV ratio was not associated with MAP in their diabetic group, but it was associated with brachial and central pulse pressure, central augmentation pressure and augmentation index. In the nondiabetic control group of their study, there was a small correlation between aortic-brachial stiffness gradient and MAP, which was no longer significant in the multivariable model. Similarly, Bia et al. [39] did not find any associations between PWV ratio and MAP in 151 hemodialysis patients.

\section{Perspective}

Aortic-brachial PWV ratio is a simple measure of arterial stiffness gradient. Indeed, PWV ratio may potentially be more promising at least in advanced aging and conditions that predispose to accelerate vascular aging, where brachial stiffness tends to decrease with age despite an increase in aortic stiffness. Even if aortic-brachial PWV ratio does not provide additional information, its lack of relationship with operating MAP makes it more appealing than crude cf-PWV. However, these observations need further external validation in different populations. In addition, the differential impact of various classes of antihypertensive drugs on the stiffness of aorta and medium-sized muscular arteries, hence their potential impact on arterial stiffness gradient needs to be elucidated.

\section{Acknowledgments}

C. Fortier holds a scholarship from the Kidney Foundation of Canada. M.P. Desjardins holds a scholarship from the Société québécoise d'hypertension artérielle through contributions from Servier and from the Canadian Institute of Health Research. M. Agharazii holds a research chair in nephrology from Université Laval.

\section{Disclosure Statement}

The author has no conflicts of interest to declare. 
Fortier et al.: Aortic-Brachial Pulse Wave Velocity Ratio: A Measure of Arterial Stiffness Gradient Not Affected by Mean Arterial Pressure

\section{References}

$\longrightarrow 1$ Willum-Hansen T, Staessen J A, Torp-Pedersen C, Rasmussen S, Thijs L, Ibsen H, Jeppesen J: Prognostic value of aortic pulse wave velocity as index of arterial stiffness in the general population. Circulation 2006;113: 664-670.

-2 Laurent S, Boutouyrie P, Asmar R, Gautier I, Laloux B, Guize L, Ducimetiere P, Benetos A: Aortic stiffness is an independent predictor of all-cause and cardiovascular mortality in hypertensive patients. Hypertension 2001; 37:1236-1241.

-3 Cruickshank K, Riste L, Anderson SG, Wright JS, Dunn G, Gosling RG: Aortic pulse-wave velocity and its relationship to mortality in diabetes and glucose intolerance: an integrated index of vascular function? Circulation 2002;106:2085-2090.

4 Blacher J, Guerin AP, Pannier B, Marchais SJ, Safar ME, London GM: Impact of aortic stiffness on survival in end-stage renal disease. Circulation 1999;99:2434-2439.

-5 Mancia G, De Backer G, Dominiczak A, Cifkova R, Fagard R, Germano G, et al; Management of Arterial Hypertension of the European Society of Hypertension, European Society of Cardiology: 2007 guidelines for the management of arterial hypertension: the task force for the management of arterial hypertension of the European Society of Hypertension (ESH) and of the European Society of Cardiology (ESC). J Hypertens 2007; 25:1105-1187.

6 Utescu MS, Couture V, Mac-Way F, De Serres SA, Marquis K, Lariviere R, Desmeules S, Lebel M, Boutouyrie P, Agharazii M: Determinants of progression of aortic stiffness in hemodialysis patients: a prospective longitudinal study. Hypertension 2013;62:154-160.

7 Fortier C, Sidibe A, Desjardins M P, Marquis K, De Serres S A, Mac-Way F, Agharazii M: Aortic-brachial pulse wave velocity ratio: a blood pressure-independent index of vascular aging. Hypertension 2017;69:96-101.

-8 Cameron JD, Bulpitt CJ, Pinto ES, Rajkumar C: The aging of elastic and muscular arteries: a comparison of diabetic and nondiabetic subjects. Diabetes Care 2003;26:2133-2138.

-9 Spronck B, Heusinkveld MH, Vanmolkot FH, RoodtJO, Hermeling E, Delhaas T, Kroon AA, Reesink KD: Pressuredependence of arterial stiffness: potential clinical implications. J Hypertens 2015;33:330-338.

10 Hermeling E, Hoeks AP, Winkens MH, Waltenberger JL, Reneman RS, Kroon AA, Reesink KD: Noninvasive assessment of arterial stiffness should discriminate between systolic and diastolic pressure ranges. Hypertension 2010;55:124-130.

11 Wolinsky H, Glagov S: Structural basis for the static mechanical properties of the aortic media. Circ Res 1964; 14:400-413.

12 Armentano RL, Levenson J, Barra JG, Fischer EI, Breitbart GJ, Pichel RH, Simon A: Assessment of elastin and collagen contribution to aortic elasticity in conscious dogs. Am J Physiol 1991;260:H1870-H1877.

13 Dobrin PB, Rovick AA: Influence of vascular smooth muscle on contractile mechanics and elasticity of arteries. Am J Physiol 1969;217:1644-1651.

14 Dobrin PB: Mechanical behavior of vascular smooth muscle in cylindrical segments of arteries in vitro. Ann Biomed Eng 1984;12:497-510.

15 Cattell MA, Anderson JC, Hasleton PS: Age-related changes in amounts and concentrations of collagen and elastin in normotensive human thoracic aorta. Clin Chim Acta 1996;245:73-84.

-16 Horinaka S, Yabe A, Yagi H, Ishimura K, Hara H, Iemura T, Ishimitsu T: Cardio-ankle vascular index could reflect plaque burden in the coronary artery. Angiology 2011;62:401-408.

$\checkmark 17$ Nakamura K, Tomaru T, Yamamura S, Miyashita Y, Shirai K, Noike H: Cardio-ankle vascular index is a candidate predictor of coronary atherosclerosis. Circ J 2008;72:598-604.

18 Korkmaz L, Adar A, Korkmaz AA, Erkan H, Agac MT, Acar Z, Kurt IH, Akyuz AR, Celik S: Atherosclerosis burden and coronary artery lesion complexity in acute coronary syndrome patients. Cardiol J 2012;19:295-300.

19 Park HE, Choi SY, Kim MK, Oh BH: Cardio-ankle vascular index reflects coronary atherosclerosis in patients with abnormal glucose metabolism: assessment with 256 slice multi-detector computed tomography. J Cardiol 2012;60:372-376.

20 Ibata J, Sasaki H, Kakimoto T, Matsuno S, Nakatani M, Kobayashi M, Tatsumi K, Nakano Y, Wakasaki H, Furuta H, Nishi M, Nanjo K: Cardio-ankle vascular index measures arterial wall stiffness independent of blood pressure. Diabetes Res Clin Pract 2008;80:265-270.

-21 Takenaka T, Hoshi H, Kato N, Kobayashi K, Takane H, Shoda J, Suzuki H: Cardio-ankle vascular index to screen cardiovascular diseases in patients with end-stage renal diseases. J Atheroscler Thromb 2008;15:339-344.

-22 Shirai K, Hiruta N, Song M, Kurosu T, Suzuki J, Tomaru T, Miyashita Y, Saiki A, Takahashi M, Suzuki K, Takata M: Cardio-ankle vascular index (CAVI) as a novel indicator of arterial stiffness: theory, evidence and perspectives. J Atheroscler Thromb 2011;18:924-938.

-23 Hayashi K, Handa H, Nagasawa S, Okumura A, Moritake K: Stiffness and elastic behavior of human intracranial and extracranial arteries. J Biomech 1980;13:175-184.

24 Spronck B, Avolio AP, Tan I, Butlin M, Reesink KD, Delhaas T: Arterial stiffness index beta and cardio-ankle vascular index inherently depend on blood pressure but can be readily corrected. J Hypertens 2017;35: 98-104.

25 Shimizu K, Yamamoto T, Takahashi M, Sato S, Noike H, Shirai K: Effect of nitroglycerin administration on cardio-ankle vascular index. Vasc Health Risk Manag 2016;12:313-319. 
Fortier et al:: Aortic-Brachial Pulse Wave Velocity Ratio: A Measure of Arterial Stiffness Gradient Not Affected by Mean Arterial Pressure

26 Yamamoto T, Shimizu K, Takahashi M, Tatsuno I, Shirai K: The effect of nitroglycerin on arterial stiffness of the aorta and the femoral-tibial arteries. J Atheroscler Thromb 2017, Epub ahead of print.

27 Agharazii M, Fortier C, Desjardins MP, Marquis K, Mac-Way F, Desmeules S, De Serres SA: Regional vascular stiffness in response to nitroglycerin and their impact on arterial stiffness gradient. J Hypertens 2016;34:e8.

-28 Avolio AP, Chen SG, Wang RP, Zhang CL, Li MF, O’Rourke MF: Effects of aging on changing arterial compliance and left ventricular load in a northern Chinese urban community. Circulation 1983;68:50-58.

29 Bortolotto LA, Hanon O, Franconi G, Boutouyrie P, Legrain S, Girerd X: The aging process modifies the distensibility of elastic but not muscular arteries. Hypertension 1999;34:889-892.

-30 Mitchell GF, Parise H, Benjamin EJ, Larson MG, Keyes MJ, Vita JA, Vasan RS, Levy D: Changes in arterial stiffness and wave reflection with advancing age in healthy men and women: the Framingham Heart Study. Hypertension 2004; 43:1239-1245.

-31 McEniery CM, Yasmin, Hall IR, Qasem A, Wilkinson IB, Cockcroft JR, Investigators A: Normal vascular aging: differential effects on wave reflection and aortic pulse wave velocity: the Anglo-Cardiff Collaborative Trial (ACCT). J Am Coll Cardiol 2005;46:1753-1760.

-32 Hashimoto J, Ito S: Central pulse pressure and aortic stiffness determine renal hemodynamics: pathophysiological implication for microalbuminuria in hypertension. Hypertension 2011;58:839-846.

-33 Tarumi T, Shah F, Tanaka H, Haley AP: Association between central elastic artery stiffness and cerebral perfusion in deep subcortical gray and white matter. Am J Hypertens 2011;24:1108-1113.

-34 Mitchell GF, van Buchem MA, Sigurdsson S, Gotal JD, Jonsdottir MK, Kjartansson O, Garcia M, Aspelund T, Harris TB, Gudnason V, Launer LJ: Arterial stiffness, pressure and flow pulsatility and brain structure and function: the Age, Gene/Environment Susceptibility - Reykjavik study. Brain 2011;134:3398-3407.

-35 Fortier C, Mac-Way F, Desmeules S, Marquis K, De Serres SA, Lebel M, Boutouyrie P, Agharazii M: Aorticbrachial stiffness mismatch and mortality in dialysis population. Hypertension 2015;65:378-384.

-36 Niiranen TJ, Kalesan B, Larson MG, Hamburg NM, Benjamin EJ, Mitchell GF, Vasan RS: Aortic-brachial arterial stiffness gradient and cardiovascular risk in the community: the Framingham Heart Study. Hypertension 2017;69:1022-1028.

-37 Picone DS, Schultz MG, Climie RE, Srikanth V, Sharman JE: Aortic-to-brachial stiffness gradient and kidney function in type 2 diabetes. J Hypertens 2016;34:1132-1139.

-38 Lee YB, Jeong SW, Rhee MY, Leem CH: Aorta-to-arm pulse wave transit time ratio: better prediction of coronary artery disease and stroke than pulse wave velocity. Int J Cardiol 2016;204:1-3.

-39 Bia D, Valtuille R, Galli C, Wray S, Armentano R, Zocalo Y, Cabrera-Fischer E: Aortic-radial pulse wave velocity ratio in end-stage renal disease patients: association with age, body tissue hydration status, renal failure etiology and five years of hemodialysis. High Blood Press Cardiovasc Prev 2017;24:37-48.

$\checkmark 40$ McEniery CM, Wilkinson IB, Avolio AP: Age, hypertension and arterial function. Clin Exp Pharmacol Physiol 2007;34:665-671. 